\section{Case Report}

Journal of Epilepsy Research pISSN 2233-6249 / elSSN 2233-6257

Received March 3, 2015 Accepted April 28, 2015

Corresponding author: Dae-Won Seo Department of Neurology, Samsung Medical Center, Sungkyunkwan University, 81 Irwon-Ro, Gangnam-gu, Seoul 135-710, Korea

Tel. +82-2-3410-3595

Fax. +82-2-3410-0052

E-mail; daewon@skku.edu

\title{
Mesial Temporal Lobe Epilepsy in Congenital Toxoplasmosis: A Case Report
}

\author{
Woo Kyo Jeong ${ }^{1}$, Byung-Euk Joo ${ }^{1,2}$, Ji-hye Seo ${ }^{1,2}$, Jun Kyu Mun ${ }^{1}$, Juhyeon Kim , Dae-Won Seo ${ }^{1,2}$ \\ ${ }^{1}$ Department of Neurology, Samsung Medical Center, Sungkyunkwan University School of Medicine, Seoul, Korea; \\ ${ }^{2}$ Neuroscience Center, Samsung Medical Center, Sungkyunkwan University School of Medicine, Seoul, Korea
}

\begin{abstract}
Toxoplasmosis is a rare disease caused by intracellular protozoan parasite, Toxoplasma gondii. Though most patients with toxoplasmosis are asymptomatic, congenital toxoplasmosis in the fetus can cause ocular involvement such as chorioretinitis and central nervous system disease including intracerebral calcification, nystagmus, hydrocephalus and microcephaly. Also, these brain lesions can cause seizure secondarily. Our patient was diagnosed with congenital toxoplasmosis, based on toxoplasma-specific serologic test with typical clinical symptoms, including chorioretinitis, nystagmus, hydrocephalus and cerebral palsy. Her brain imaging findings revealed not only the multifocal encephalomalacia, but also multifocal cerebral calcification including intracerebral calcification in left perihippocampal region. Her epileptogenic zone was defined as mesial temporal lobe including hippocampus on left side by seizure semiology, electroencephalogram and neuroimaging including single photon emission computed tomography and $18 \mathrm{~F}-$ Fluorodeoxyglucose positron-emission tomography. Her seizures were refractory to multiple anti-epileptic drugs. We report a patient with congenital toxoplasmosis who showed intractable mesial temporal lobe epilepsy. (2015;5:25-28)
\end{abstract}

Key words: Congenital toxoplasmosis, Temporal lobe epilepsy, Intracerebral calcification

\section{Introduction}

Toxoplasmosis is caused by the obligate intracellular protozoan parasite, Toxoplasma gondii, for which preferred primary host is a cat and human may become infected as an intermediate host. ${ }^{1}$ Most people with toxoplasmosis are asymptomatic. However, if a pregnant woman is infected, the parasite can enter the fetal circulation through placenta and may cause congenital toxoplasmosis in the fetus. Congenital toxoplasmosis may present with a broad spectrum of clinical manifestations, ranging from mild chorioretinitis to severe central nervous system (CNS) involvement, including hydrocephalus, mental retardation, seizures and intracerebral calcifications. ${ }^{2,3}$

Seizure is one of the main presenting symptoms in patients with CNS infection of congenital acquired toxoplasmosis and many patients with congenital toxoplasmosis took anti-epileptic drugs (AED) constantly. However, there have been few reports of detailed description of seizure semiology and besides few reported cases of a patient with congenital toxoplasmosis who was suffering from temporal lobe epilepsy with hippocampal sclerosis (HS), which is one of the most common epileptic syndromes and is considered medically intractable but surgically remediable in any causes. ${ }^{4,5}$

Hereby we described a patient with congenital toxoplasmosis who showed intractable mesial temporal lobe epilepsy.

\section{Case}

A 34-year-old left handed Arabian female was presented with our hospital with medically intractable seizure. The patient was born preterm with fetal infection by Toxoplasma gondii. She had hydrocephalus and right ventriculoperitoneal (VP) shunting was performed at 1 month old. She experienced convulsive seizures since 8 months old. Her development was delayed than other children. She started walk at 3 years and right hemiparesis was observed. She also complained of decreased visual acuity in both eyes. On past history, she had no history of febrile seizure, metabolic disease or head trauma and her family also had no history of seizure.

In a neurological examination, contracture and weakness of right arm and leg was found compatible with cerebral palsy. Her mental status was evaluated using Mini-Mental State Examination with the help of a translator and her score was 22, suggesting memory im- 
pairment and inattention. In ophthalmologic evaluation for visual impairment, macular inferior chorioretinal scar in right eye and large gray-whitish retinal necrotic lesion on posterior pole in left eye was found compatible with chorioretinitis which was characteristic finding usually seen in the patients with congenital toxoplasmosis (Fig. $1 A, B)$. In serologic test using enzyme linked fluorescent assay, toxoplasma-specific lgG test was positive and IgM test was negative, suggesting chronic phase of T. gondii infection. ${ }^{6}$

The patient and her mother reported that her seizures were started with psychic aura such as anxiety initially and then secondarily gener-
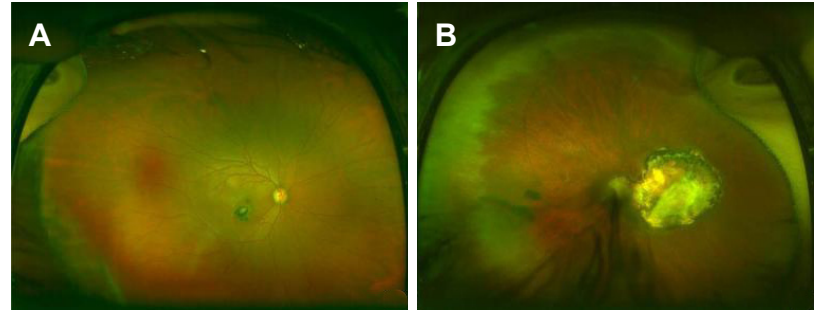

Figure 1. Fundus photography in a patient with congenital toxoplasmosis (A) Image shows macular inferior chorioretinal scar in right eye. (B) Image reveals large gray-whitish retinal necrotic lesion on posterior pole in left eye.
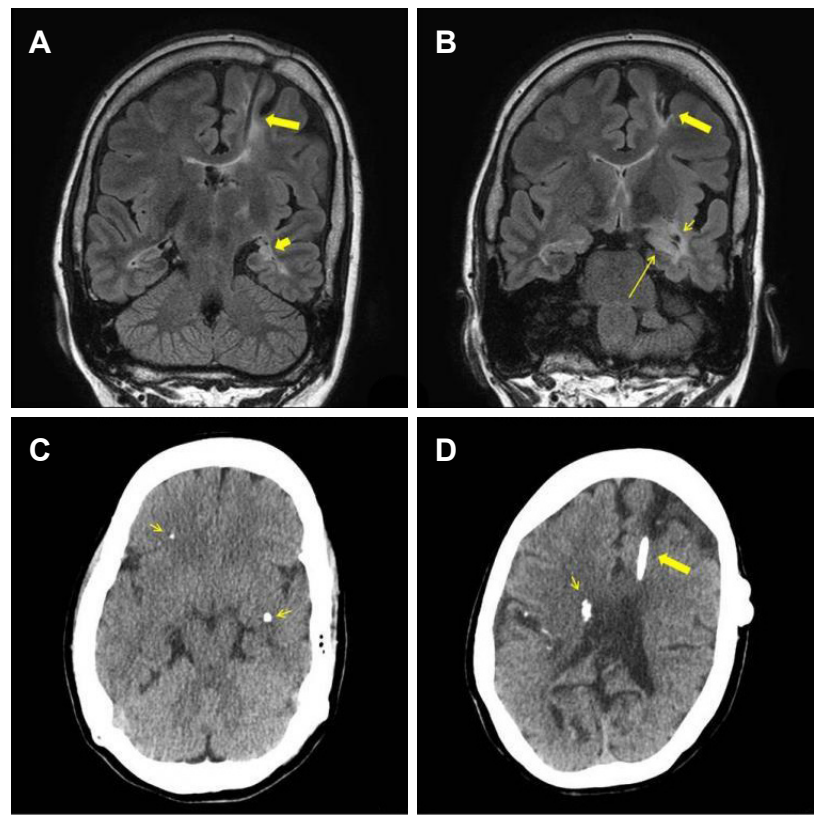

Figure 2. Brain MRI and CT. (A) and (B) Coronal FLAIR MRI shows multifocal encephalomalacic change related to VP shunt (wide long arrow), left hippocampal sclerosis (wide short arrow) and left amygdala hypertrophy (thin long arrow) and intracerebral calcification (thin short arrow) adjacent to hippocampus. (C) and (D) Axial CT demonstrates multiple intracerebral calcification in left perihippocampal region, periventricular and subcortical white matter. $C T$, computed tomography; MRI, magnetic resonance imaging; FLAIR, fluid attenuated inversion recovery; VP, ventriculoperitoneal. alized seizure with right eyeball deviation and head version. In the beginning, her seizures occurred one to three times a month. However the frequency of her seizure were gradually increased over time, and though the dose of AED was increased, the frequency of seizure was not reduced. Finally, she had the polytherapy of AEDs including levetiracetam, lamotrigine and topiramate, but seizures were still poorly controlled. For further evaluation and management of her seizure, she was referred to our epilepsy clinic.

She underwent 3.0T high resolution brain magnetic resonance imaging (MRI). On brain MRI, multifocal encephalomalacic change related to VP shunt, left HS and left amygdala hypertrophy, and multifocal parenchymal calcification including left perihippocampal region were observed. The parenchymal calcification was confirmed again by brain computed tomography (CT), which was compatible with congenital toxoplasmosis.

Video/electroencephalogram (EEG) monitoring was performed for 6 days. During video-EEG monitoring, 5 complex partial seizures (CPS) were observed, and 2 of those 5 CPS were led to generalized tonic clonic (GTC) seizure. All CPSs were started with psychic aura such as anxiety, and dialeptic seizure was followed. In secondary GTC seizures, tonic posture of right arm and leg and head version to right side were accompanied.

On EEG, all ictal events were started on left mesial temporal area. The rhythmic delta activity was observed initially on left temporal
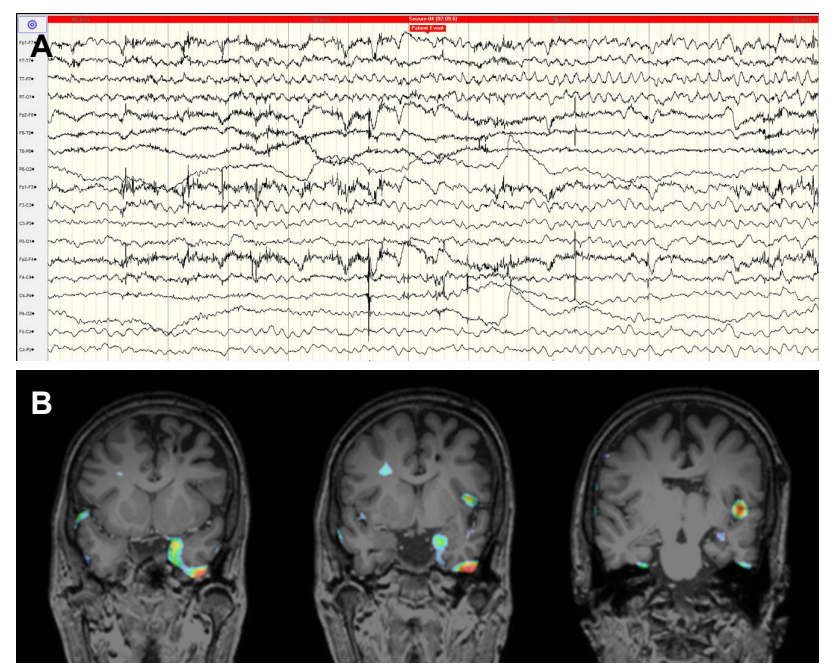

Figure 3. Ictal EEG and ictal SPECT. (A) The ictal EEG recording demonstrates that her seizure starts with delta waves in left temporal area which build up into rhythmic theta waves spreading into left hemisphere. (B) SISCOM shows the ictal hyperperfusion zone in left temporal areas. EEG, electroencephalogram; SPECT, single photon emission computed tomography; SISCOM, Substracted ictal SPECT coregistered to MRI. 
area, and then led to rhythmic theta waves in the same area, spreading into left hemisphere. Intermittent slowing in left temporal area was also seen in interictal EEG monitoring and no epileptiform discharges were seen.

She underwent ictal and interictal 99m-Technetium (Tc) hexamethylpropyleneamineoxime single photon emission computed tomography (HMPAO-SPECT). Ictal hyperperfusion was seen in the bilateral frontotemporal areas, more pronounced on the left side. Interictal SPECT, taken after at least 24 hour seizure freedom, showed hypoperfusion in the left frontotemporal area. Substracted ictal SPECT coregistered to MRI (SISCOM) shows the ictal hyperperfusion zone in left mesial temporal areas. Brain ${ }^{18} \mathrm{~F}$-Fluorodeoxyglucose positronemission tomography (FDG-PET) demonstrated focal hypermetabolism in left medial temporal cortex compatible with postictal status and hypometabolism in the left fronto-parieto-temporal cortex. Wada test was performed to determinate the lateralization of hemispheric speech and memory dominance and it revealed right hemisphere language and memory dominance, predictive of good outcome after left anteromesial temporal resection. Considering that her seizure was medically intractable and epileptogenic focus was determined to be in left temporal lobe by presurgical evaluation, we decided to perform epilepsy surgery with left anteromesial temporal resection including calcified lesion to achieve seizure control.

\section{Discussion}

This is a case report of medically intractable mesial temporal lobe epilepsy in a patient with congenital toxoplasmosis, diagnosed by toxoplasma-specific serologic test with typical symptoms and signs including chorioretinitis, growth retardation, cerebral palsy and hydrocephalus.

In patients with congenital toxoplasmosis, it is well known that infection may invade tissues in central nervous system and can cause diffuse and multifocal parenchymal necrosis forming calcifications, which are main characteristics of this disease. In addition, the distribution and severity of calcifications vary according to the date of maternal infection and also correlate with neurologic signs and symptoms. ${ }^{7,8}$ In our case, multifocal small parenchymal calcifications including a calcified nodule in left perihippocampal area was found in brain imaging findings, and HS and amygdala hypertrophy on left temporal lobe were also observed. Our patient presented predominantly dialeptic seizure accompanied by psychic aura during ictal events, which was a commonly observed finding in TLE. TLE was con- firmed with ictal and interictal EEG findings, and neuroimaging including SPECT and SISCOM.

$\mathrm{HS}$ is the most common finding in mesial temporal lobe epilepsy, closely associated with medically intractable epilepsy. Though the cause of HS remains unknown, many hypotheses including febrile seizures, genetic susceptibility or acquired damage such as inflammation, are considered. Also, it is well known that recurrent seizures may cause hippocampal neuronal damage in animals similar to hippocampal sclerosis, which may be considered as a mechanism in patients with dual pathology, coexistence of HS with extrahippocampal lesion. ${ }^{5,9}$ Our patient had no history of febrile seizure and no family history of epilepsy, but on brain imaging study, HS and amygdala hypertrophy were demonstrated concomitant with a calcified nodule in perihippocampal region by congenital toxoplasmosis. In this case, the pathogenesis of $H S$, which may be the cause of the TLE, can be considered by several different mechanisms. One of possible mechanism of HS is that seizure due to a calcified nodule constitutes an initial precipitating illness, leading to the development of HS. The kindling phenomenon of epilepsy is known as a process in which repeated low-level stimuli result in seizure development until a plateau is reached. This phenomenon is one of well-known model of epileptogenesis and also has been closely related to mesial temporal lobe epilepsy in respect of inducing progressive neuronal loss in hippocampal formation by repetitive seizure. ${ }^{10,11}$ This hypothesis has been suggested in several case reports of other disease such as neurocysticercosis. Another possible mechanism is that hippocampus may be impacted by the inflammatory reaction and resulting gliosis around the calcified nodule. There was a case report of a patient with neurocysticercosis and intractable mesial temporal lobe epilepsy. ${ }^{12}$ In that case, calcified mass near the hippocampus and abundant corpora amylacea were found, suggesting that degenerating cysticercus induced inflammatory injury to hippocampus. Of course, both mechanisms could contribute to HS in same patient. Also, the possibility that a calcified nodule by congenital toxoplasmosis and HS may coexist purely by chance cannot be excluded.

TLE accompanied with HS is usually medically intractable, and hippocampal damage is known to progress in patient with medically intractable TLE. So it needs to be very careful when managing with seizure in patients with congenital toxoplasmosis, accompanied with calcification in the perihippocampal area. Comprehensive evaluation including video-EEG monitoring, SPECT, FDG-PET and MRI might be needed for defining epilepsy and localizing epileptogenic foci accurately, and be necessary to consider the surgical treatment for man- 
aging seizure if possible.

In Korea, there have been many surveys on seroprevalence of toxoplasmosis, which are in the range from 1.9 to $7.7 \%$ in the 1980 90s. ${ }^{13-15}$ These result was significantly lower than those of the other endemic countries of 30-70\%. ${ }^{16}$ However, with increase of the number of domestic cats and change of eating habits to meat preference, seroprevalence of toxoplasmosis in Korea has increased to 13$25 \% .{ }^{17,18}$ Furthermore, considering high prevalence of $T$. gondii in the stray cats in Korea, ${ }_{1}^{19,20}$ these data imply that the number of patients with congenital toxoplasmosis like our case may increase, thus careful prenatal and neonatal monitoring for congenital toxoplasmosis is necessary and essential in Korea.

In our opinion, the association of congenital toxoplasmosis with mesial temporal lobe epilepsy has been poorly reported so far, and also patients with congenital toxoplasmosis showing seizure who underwent video EEG monitoring and appropriate neuroimaging study were reported rarely.

We report a case with medically intractable mesial temporal lobe epilepsy with congenital toxoplasmosis, in which presented both HS and a calcified nodule in perihippocampal area. There may be several hypotheses for the observed HS in patients with congenital toxoplasmosis, and limits to define a cause-effect relationship between the two conditions among several mechanisms. When managing seizure disorder in patients with congenital toxoplasmosis, neurologists should be aware of this association, and the surgical treatment should be considered for managing seizure when patients with congenital toxoplasmosis presented with medically intractable TLE and a calcified nodule in perihippocampal area.

\section{Acknowledgments}

There is no funding and financial or material support.

\section{References}

1. Kaye A. Toxoplasmosis: diagnosis, treatment, and prevention in congenitally exposed infants. J Pediatr Health Care 2011;25:355-64.

2. Kravetz JD, Federman DG. Toxoplasmosis in pregnancy. Am J Med 2005;118:212-6.

3. Jones J, Lopez A, Wilson M. Congenital toxoplasmosis. Am Fam Physician 2003;67:2131-8.

4. Wiebe S, Blume WT, Girvin JP, Eliasziw M, Effectiveness, Efficiency of
Surgery for Temporal Lobe Epilepsy Study G. A randomized, controlled trial of surgery for temporal-lobe epilepsy. N Eng/ J Med 2001;345: 311-8.

5. Engel J, Jr. Introduction to temporal lobe epilepsy. Epilepsy Res 1996; 26:141-50.

6. Montoya JG. Laboratory diagnosis of Toxoplasma gondii infection and toxoplasmosis. J Infect Dis 2002;185 Suppl 1:S73-82.

7. Lago EG, Baldisserotto $M$, Hoefel Filho JR, Santiago $D$, Jungblut $R$. Agreement between ultrasonography and computed tomography in detecting intracranial calcifications in congenital toxoplasmosis. Clin Radiol 2007;62:1004-11.

8. Diebler C, Dusser A, Dulac 0. Congenital toxoplasmosis. Clinical and neuroradiological evaluation of the cerebral lesions. Neuroradiology 1985;27:125-30.

9. Cendes F, Cook MJ, Watson C, et al. Frequency and characteristics of dual pathology in patients with lesional epilepsy. Neurology 1995;45: 2058-64.

10. Goddard GV. Development of epileptic seizures through brain stimulation at low intensity. Nature 1967;214:1020-1.

11. Bertram E. The relevance of kindling for human epilepsy. Epilepsia 2007;48 Suppl 2:65-74.

12. Chung CK, Lee SK, Chi JG. Temporal lobe epilepsy caused by intrahippocampal calcified cysticercus: a case report. J Korean Med Sci 1998;13:445-8.

13. Choi WY, Nam HW, Youn JH, Kim WS, Kim WK. Toxoplasma antibody titers by indirect latex agglutination test in patients of Kangnam St. Mary's Hospital and Cheju Medical Center. Kisaengchunghak Chapchi 1989;27:171-5.

14. Choi WY, Nam HW, Youn $J$, et al. Detection of antibodies in serum and cerebrospinal fluid to Toxoplasma gondii by indirect latex agglutination test and enzyme-linked immunosorbent assay. Kisaengchunghak Chapchi 1992;30:83-90.

15. Song KJ, Shin JC, Shin HJ, Nam HW. Seroprevalence of toxoplasmosis in Korean pregnant women. Korean J Parasitol 2005;43:69-71.

16. Tenter AM, Heckeroth AR, Weiss LM. Toxoplasma gondii: from animals to humans. Int J Parasitol 2000;30:1217-58.

17. Hong SJ, Chong CK, Lee $K$, et al. Maintained seroprevalence of toxoplasmosis among the residents of Jeju island, Korea. Korean J Parasitol 2011:49:309-11.

18. Yang Z, Cho PY, Ahn SK, et al. A surge in the seroprevalence of toxoplasmosis among the residents of islands in Gangwha-gun, Incheon, Korea. Korean J Parasitol 2012;50:191-7.

19. Kim HY, Kim YA, Kang $S$, et al. Prevalence of Toxoplasma gondii in stray cats of Gyeonggi-do, Korea. Korean J Parasito/ 2008;46:199-201.

20. Lee SE, Kim NH, Chae HS, et al. Prevalence of Toxoplasma gondii infection in feral cats in Seoul, Korea. J Parasitol 2011;97:153-5. 